\title{
Don Quijote. Revista Mensual de Arte (1908-1911): estudio inicial de la cultura literaria en Puebla durante la primera década del siglo XX
}

Don Quijote. Revista Mensual de Arte (1908-1911): initial study of Puebla's literary culture in 20th century first decade

\author{
Alejandro Palma Castro \\ ORCID: 0000-0002-8414-7602 \\ alejandro.palmaffyl@gmail.com
}

Benemérita Universidad Autónoma de Puebla, México

Ruth Miraceti Rojas Jiménez

ORCID: 0000-0001-6112-1541

ruthmiraceti.rojas@hotmail.com

Benemérita Universidad Autónoma de Puebla, México

Resumen:

Don Quijote. Revista Mensual de Arte fue una revista editada por alumnos del Colegio del Estado de Puebla y resulta un testimonio fundamental para comprender los cambios tanto culturales como políticos y sociales que se fueron gestando en dicha ciudad durante la primera década del siglo XX. A través del estudio de su índice, se establece una relación entre este campo literario y el campo político local y nacional ante el inminente estallido de la Revolución mexicana. El estudio se enmarca en la noción de campo literario que ha establecido Pierre Bourdieu. El análisis de estas relaciones permite recuperar personalidades de la época así como dar cuenta de estéticas e ideas literarias predominantes. De igual manera, mostramos la existencia de una red de revistas culturales de distintos puntos de la provincia 
mexicana, que hacía frente al centralismo cultural de la Ciudad de México. Como resultado de la investigación, se invita a continuar con este tipo de estudios que permiten reconocer el amplio y diverso panorama de la literatura mexicana hacia comienzos del siglo XX.

Palabras clave:

Revista Don Quijote, Colegio del Estado de Puebla, índice de revista, literatura mexicana y provincia.

Abstract.

Don Quijote. Revista Mensual de Arte was a magazine published by students of the Colegio del Estado de Puebla during the first decade of $20^{\text {th }}$ century. Through the study of its index, we can document the main concerns and changes, in both literary and political fields, before the imminent outbreak of the Mexican Revolution. Using Pierre Bourdieu's notion of literary field we trace the relation of the magazine's main ideas and the local and national politics of that time. The analysis of these relationships makes it possible to show the main personalities, aesthetic and literary ideas surrounding the decade. Through that, we establish a network among periodical publications in different cities of Mexico, which confronted the cultural centralism in Mexico City. As a result of this investigation we call for similar studies that could describe the wide and diverse literary scope in Mexico at the beginning of the $20^{\text {th }}$ century.

Keywords:

Don Quijote magazine, Colegio del Estado de Puebla, Magazine index, Mexican regional literature.

DOI: https://doi.org/10.36798/critlit.vi21.330

Recibido: 26 de septiembre de 2019 Aceptado: 25 de febrero de 2020 
Don Quijote. Revista Mensual de Arte fue una revista editada por alumnos del Colegio del Estado de Puebla que, a pesar de su corta existencia, ${ }^{1}$ resulta un testimonio fundamental para comprender los cambios tanto culturales como políticos y sociales que se fueron gestando en dicha ciudad durante la primera década del siglo XX. Sus principales artífices, Rafael Cabrera y Alfonso G. Alarcón, jóvenes estudiantes de medicina, buscaban brindar un sustento intelectual a los cambios políticos y sociales que acontecían. Ambos eran simpatizantes maderistas antirreeleccionistas que promovían y participaban en actividades políticas a favor de Francisco I. Madero, como su recepción en Puebla en calidad de candidato a la presidencia. ${ }^{2}$ Posteriormente, tras el estallido de la lucha armada, diversas circunstancias estrecharon el vínculo de ambos con el Ateneo de la Juventud Mexicana (Pedro Henríquez Ureña, Antonio Caso, Alfonso Reyes, Julio Torri, José Vasconcelos), primero con Reyes, pese a existir un capítulo previo de desacuerdo que explicaremos más adelante.

Dada la preeminencia posterior que lograrían estos personajes en la vida universitaria y mexicana - Cabrera como poeta modernista y diplomático de primer orden, ${ }^{3}$ y Alarcón como el segundo rector de la Universidad de Puebla (1938-1941) y médico reconocido

${ }^{1}$ Existe una segunda época de la revista que no vamos a retomar para este estudio y que va de 1932 a 1935. Nos parece que esta publicación, aunque animada por una nueva generación de estudiantes del Colegio del Estado, según lo consigna Enrique Cordero y Torres en Historia del periodismo en Puebla (1820-1946), pp. 368-370, no representa como tal una segunda época, de no ser porque a decir del mismo Cordero y Torres: "Posiblemente el prestigio que esta publicación tuvo en su primera época fue factor de importancia para la buena acogida en su reaparición” (368). Por lo demás, parece atender a otro contexto político, social y cultural que hace difícil sostener la idea de continuidad de un proyecto cultural.

${ }^{2}$ Humberto Sotelo Mendoza ha revivido este capítulo de la historia universitaria en un número dedicado exprofeso para Tiempo Universitario.

${ }^{3} \mathrm{Al}$ respecto puede consultarse "Un ateneísta olvidado" de José Carlos Blázquez, quizás la semblanza y estudio más completo que se haya publicado hasta ahora como parte de una investigación amplia sobre la obra del poeta, médico, diplomático y adepto al ocultismo. 
nacionalmente por su investigación ${ }^{4}$ - , resulta fundamental conocer su historia, la cual se remonta a la revista Don Quijote. Ambos, de vena poética, concibieron una revista literaria de corte moderno, es decir, que hiciera frente al progreso fijado por el positivismo y determinismo caducos, para, en su lugar, acoger a una nueva generación de jóvenes dispuestos a asimilar los cambios sociales no solamente del país, sino del mundo, a través de la lectura y la escritura.

A partir de la consignación del índice de la revista ${ }^{5}$ fue posible obtener datos que amplían el escaso conocimiento que existe sobre la literatura que se escribía y leía en Puebla en los albores del siglo $\mathrm{XX}$. Así, se reconoció el tránsito que hubo de diversos escritores decimonónicos hacia la conformación de una nueva cultura y sociedad, aquella denominada moderna. Por otro lado, se recuperó el registro de distintas personalidades nacionales y extranjeras que colaboraron a lo largo de la publicación mensual para dar cuenta de estéticas e ideas literarias predominantes. Al estudiar dicha nómina resultó revelador el reconocimiento de una red de revistas culturales de diversos puntos de la provincia mexicana, donde incluso se pugnaba por un provincialismo frente al centralismo cultural de la Ciudad de México. ${ }^{6}$

${ }^{4}$ Para la importancia de Alarcón como promotor de la Universidad de Puebla en su organización y planes de estudio, se puede revisar el trabajo de Jesús Márquez Carrillo, "Universidad y política regional en Puebla, México: 1934-1990". Sobre su trabajo como médico e investigador se encuentra una nota histórica a cargo de Silvestre Frenk: "Las originales aportaciones del doctor Alfonso G. Alarcón (1884-1953) al conocimiento de la disfunción gastroesofágica en el lactante mayor".

${ }^{5}$ Esta labor ha sido realizada por Ruth M. Rojas Jiménez como parte de su trabajo de investigación en el Doctorado en Literatura Hispanoamericana de la Benemérita Universidad Autónoma de Puebla. En este estudio no solo se retoman los índices, sino que también se consultaron la revista y sus textos de primera mano, pues no existe más información o estudios sobre la revista.

${ }^{6}$ A diferencia de los estudios que se han realizado sobre revistas literarias producidas en la Ciudad de México a principios del siglo XX, las revistas de provincia permanecen casi inexploradas. El índice de Don Quijote permitió reconocer el intercambio y red que se constituyó con publicaciones similares en otros 
Finalmente, a pesar de ser una revista dedicada al arte, las diversas tomas de posición ${ }^{7}$ permiten establecer una relación entre este campo literario y el campo político local y nacional ${ }^{8}$ ante el inminente estallido de la Revolución mexicana. En este artículo se desarrollará la descripción general de dichos datos a manera de introducción para que, a partir del establecimiento del índice de esta revista y la mención de algunos de sus textos, así como de su relación con otras publicaciones afines de la misma época, se alienten más estudios para un conocimiento profundo de lo que significó la entrada a la primera década del siglo XX en Puebla y el país para varios escritores y artistas.

En un amplio y documentado estudio publicado en la Revista Iberoamericana sobre la revista literaria en Hispanoamérica, ${ }^{9}$ John E. Englekirk considera que "La prensa periódica constituye una fuente de materia prima imprescindible para un más profundo conocimiento de la literatura hispanoamericana" (9), pues "para todo investigador la revista literaria propiamente dicha se ha presentado como órgano oficial, o portavoz, de muchas generaciones o promociones estéticas que han caracterizado la evolución de las letras en las Américas" (9). Es así como el estudio de dichas publicacio-

lugares del país. Entre la más mencionadas se encuentran: Fausto, en Cuautla, Morelos; Kalendas, de Lagos de Moreno, Jalisco; Alborada, también de Lagos de Moreno; Capullos, de San Luis Potosí.

${ }^{7}$ Concepto retomado de la teoría de campos de Pierre Bourdieu, a través de la cual explica que el campo de tomas de posición "es el conjunto estructurado de las manifestaciones de los agentes sociales comprometidos en el campo —obras literarias o artísticas, evidentemente, pero también actos y discursos políticos, manifiestos o polémicas, etc._" ("El campo literario..." 4).

${ }^{8}$ Bourdieu plantea que los campos, vistos como el espacio en donde se gestan distintas luchas que buscan transformar el campo de fuerzas en donde se posicionan distintos actores, se relacionan unos con otros.

${ }^{9}$ Dicho estudio resulta de importancia dado que coincide con otros dos que a la postre resultaron más conocidos: el de Boyd G. Carter, Las revistas literarias de Hispanoamérica; y el de Sturgis E. Leavitt, Revistas hispanoamericanas. Índice bibliográfico 1843-1935. No obstante, ninguno consigna la revista Don Quijote, algo que sí hace el trabajo de Englekirk. 
nes ha llevado, con frecuencia, a una ampliación del conocimiento sobre una época o corriente literaria, e incluso a la reconsideración de diversos juicios previamente establecidos. En el caso que nos ocupa, la literatura escrita en Puebla durante los albores del siglo $\mathrm{XX}$, esto no puede ser más evidente.

Existe poco conocimiento sobre dicho periodo en Puebla dada la escasa publicación y preservación de libros publicados, frente a otros factores como la atención desigual al proceso modernizador de inicios del siglo XX que ocurrió en la capital mexicana. ${ }^{10}$ Pero a partir de la publicación Don Quijote se reconocen, desde algunas de las contribuciones, las inquietudes que existían entre la juventud del momento, así como los principales debates que se libraban en materia de cultura y política. Esto es posible debido a que, a pesar de que la revista tuvo una corta vida si se la compara con otras publicaciones que han trascendido en la historiografía literaria, en ella se agrupó un destacado sector de la población universitaria del Colegio del Estado, que buscaba una nueva expresión literaria, además de ser políticamente antirreeleccionista. Al respecto de la revista, Antonio Esparza escribe: "A su amparo se inició una labor fecunda, que si bien no maduró todos los frutos que de ella se esperaban, sí fue una atalaya de luz en el ambiente intelectual de su época" (93).

${ }^{10}$ Algunos de los principales estudios sobre la literatura publicada en Puebla son los dos tomos de Puebla, una literatura del dolor (1610-1994), de Pedro Ángel Palou García; Eslabones para una historia literaria de Puebla durante el siglo XIX, de Alejando Palma Castro y Alicia V. Ramírez Olivares; Antes de dar vuelta. La poesía que leían los poblanos 1901 a 1922, de Guadalupe Prieto; el texto "Las letras universitarias del siglo XX", de Antonio Esparza, miembro fundador de la revista Cauce de 1945, donde establece la importancia de las instituciones educativas en la formación de escritores; la investigación de Joel Dávila Gutiérrez, "El estudio de la literatura regional poblana (1945-1995)"; o el texto "Un ateneísta olvidado", de José Carlos Blázquez. Por otra parte, con respecto a los textos históricos que han abordado el periodo de la Revolución mexicana en esta ciudad están Historia de la educación pública en Puebla, 1790-1982, de Salvador Cruz; Educación, historia y sociedad en Puebla. Raíces, tiempos, buellas, de Jesús Márquez; "Los estudiantes poblanos en 1910”, de Ana Ma. Huerta; "El Colegio del Estado de Puebla y el Primer Congreso Nacional de Estudiantes en 1910”, de Ma. de Lourdes Herrera Feria, entre otros. 
El primer número de la revista Don Quijote se publicó el sábado primero de febrero de 1908. A partir de ahí, circuló el primero de cada mes hasta 1911 y se publicaron diez números por cada tomo. El fundador y propietario fue Lorenzo Aburto, un liberal moderado, y los directores fueron Rafael Cabrera y Alfonso G. Alarcón, estudiantes del Colegio del Estado. Así mismo, figuraron Aurelio M. Aja y Gil Jiménez como Secretario de Redacción y Administrador, respectivamente. Se imprimió en "El escritorio"11, imprenta ubicada, durante 1910, en la calle Zaragoza núm. 8. La revista tenía un costo de 90 centavos por serie de diez números y de 45 centavos por cinco números, mientras que fuera de Puebla se vendía la serie de diez por un peso. El número suelto se vendía en diez centavos; el atrasado, en quince.

Como publicidad, se integraron anuncios de, por ejemplo, La Tabacalera Mexicana S. A., los cigarros Reyna Victoria, la Cervecería Cuauhtémoc, tintas y cintas para máquinas de escribir, la agencia de negocios de Leopoldo Alvarado, el gabinete de análisis químicos del profesor Manuel Ibáñez, la funeraria de Juan P. Hernández, la Librería católica de Puebla de Enrique del Moral, los corredores titulados Ricardo Rugerio y Simitrio Rugerio, la fábrica de muebles de Francisco Ramírez Daza, así como las bicicletas Swift de Ligorio López y la oftalmología de Luis G. Samaniego. En el tomo IV, número 5 , agregaron un mayor espacio para publicidad, en donde cobraban 50 centavos por inserción. ${ }^{12}$

Además de Cabrera, quien publicó en todos los números, y de Alarcón, la revista contó con la inclusión de diversos escritores del estado — la mayor parte—, así como del país y del extranjero: Felipe

${ }^{11}$ Es solo a partir del número 10 que se consigna a este taller como la imprenta de la revista, aunque es probable que haya sido impresa ahí desde el primer número.

${ }^{12}$ En el número 8 de 1908 se indica que El Heraldo de Puebla, un diario local, comienza a pagar la impresión de Don Quijote: "Los Sres. Fernando Blumenkron y Eduardo Arrioja, inteligentes editores de 'El Heraldo de Puebla,' á ejemplo del grupo de intelectuales que sostienen esta revista, nos han prometido particularmente ... contribuir al mismo fin, sufragando los gastos de papel é impresión de 'Don Quijote"' (16). Sin embargo, no queda constancia de cuánto duró dicho patrocinio. 
T. Contreras, Manuel Rivadeneyra y Palacio, José Miguel Sarmiento, Eduardo Correa, María Enriqueta (Camarillo), Rafael Serrano, Juan Sánchez Azcona, Andrés Calcáneo, Bonifacio Byrne, M. A. Silva Gandolphi, Gregorio Martínez Sierra, Santiago Rusiñol, Luis Sánchez Pontón, Francisco Escobedo, Julio Torri, Mariano Azuela, Cayetano Rodríguez Beltrán, Amado Nervo, Ernest Mourguet, Selma Lagerlöf, Gastón Rageot, Georges Grappe, Giuseppe Fanciulli, Henryk Sienkiewicz (consignado como Enrique Sienkewicks), Mikhail Yuryevich Lermontov (consignado como Mikhail Yuryvitch Lermontoff), Annie Trumbull Slosson, entre muchos más.

La revista se dividía en diferentes secciones. En "Joyas literarias" se publicaban clásicos como Garcilaso de la Vega, Luis de Góngora o Francisco Quevedo; en la "Sección Bibliográfica" se consignaban libros recién publicados y su crítica; otra sección, "Arte extranjero", incluía autores como Mourguet, Éphraïm Mikhaël, Jules Deldon, Mark Twain y Angelo Maria Tirabassi. Así mismo, contaba con apartados como "Poemas en prosa" y "Tipos callejeros", con narraciones sobre personajes picarescos, lo cual refleja un sello particular de la publicación. Como revista dedicada no solo a la literatura sino a la cultura, dio cabida a contenidos variados: crónicas, poesía, cuento, crítica, fotografía, pintura, grabado, teatro, entre otras artes, no únicamente de nacionales, sino también de extranjeros. Incluso, pueden encontrarse traducciones hechas por encargo, así como textos inéditos y exclusivos ${ }^{13}$ para la revista. También se incluían ilustraciones, retratos, reproducciones de cuadros, caricaturas y fotografías de autores como W. Gentz, Diego Morón, Leonardo Bastolfi, J. P. Hernández, Rafael Cabrera y Alfonso G. Alarcón.

Respecto a la tendencia literaria que abrazaba la revista, el editorial, consignado como "Programa" en el número prospecto (Fig. 1), es claro y preciso. Se trata de una revista realizada con "fervoroso entusiasmo por el Ideal con que nos ha nutrido nuestro hidalgo

\footnotetext{
${ }^{13}$ Son las inferencias que hemos realizado hasta ahora tras cotejar con algunas obras completas de autores como María Enriqueta Camarillo, Mariano Azuela y Julio Torri.
} 
Maestro" ("Programa”). El título de la revista obedecerá entonces, de acuerdo con nuestra interpretación, a un espíritu juvenil que busca idealizar un mundo mejor al margen de la avanzada capitalista —en el mismo programa se dice: "el ideal existe, pese a las casas empacadoras y las salchichonerías"- y el pragmatismo modernizador: "Nutridos en las máximas del más puro espejo de los caballeros andantes, le dimos una prueba inmediata del acatamiento que de sus doctrinas hacíamos, asesinando implacablemente á Sancho". ${ }^{14}$ Esta idealización del "espejo [enseñanzas] de los caballeros andantes" se manifiesta como una prolongación del romanticismo, además de fijar una orientación castiza derivada de la consagración de la novela de Miguel de Cervantes como modelo de la más alta literatura ${ }^{15}$ a través de su personaje principal.

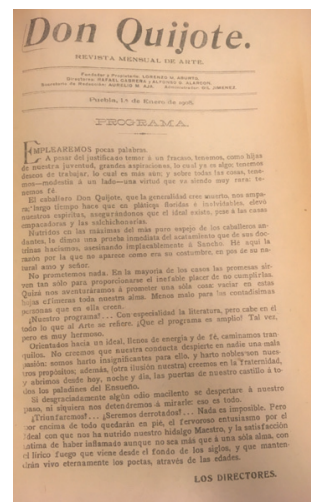

Fig. 1. Primera página del número prospecto de Don Quijote, 15 de enero de 1908. Escaneo propio tomado en la Biblioteca de la Universidad de las Américas Puebla.

${ }^{14}$ Es importante mencionar que, quizás como contraparte de esta toma de posición, surgirá en 1910 una revista de corte satírico, Sancho, la cual se abrirá a una crítica más directa contra el Colegio del Estado, algunos escritores de Don Quijote (como Alfonso G. Alarcón) el Seminario Palafoxiano y la reelección porfirista de 1910.

${ }^{15}$ Posición que coincidía con lo que el español Azorín — a quien por cierto publicaron en dos ocasiones en la revista - consolidara en 1913 bajo la denominación "Generación del 98". 
En este sentido, se buscaba generar una alternativa al predominio de la literatura francesa a lo largo de casi todo el siglo XIX en Hispanoamérica, cuya última tendencia, el decadentismo, ${ }^{16}$ fue duramente criticada por los defensores de la larga tradición literaria castellana. Don Quijote sostuvo, durante sus primeros años, una fuerte crítica contra el decadentismo y el modernismo centralista, iniciada por uno de sus fundadores, Atenedoro Monroy, quien en 1902 escribió "Valor estético de las obras de la escuela decadentista", que no solo se ganó un premio en los Juegos Florales de Puebla de ese año, sino también la animadversión de los escritores del centro a través de debates publicados en la Revista Moderna (1898-1903). ${ }^{17}$ Este texto formó parte de una serie de acciones que buscaban la visibilidad y autonomía ${ }^{18}$ de un grupo homogéneo de escritores tradicionalistas, quienes en 1907 conformaron el Ateneo de la Juventud Poblana y que, un año después, dieron pie a la creación de Don Quijote. Monroy escribe en sus primeros números, además de ser un

${ }^{16}$ Según José Mariano Leyva en Perversos y pesimistas: "A México llegó [el decadentismo] por medio de cuentos, novelas y de la Revista Moderna, la cual sirvió de cuartel general para los decadentes cuando varios ya habían sido vetados de otras publicaciones periódicas. Hablamos de los escritores José Juan Tablada y Amado Nervo —en sus estrictas juventudes—, además de Alberto Leduc —el padre de Renato-, Bernardo Couto, Jesús E. Valenzuela, Efrén Rebolledo, Ciro B. Ceballos, Rubén M. Campos, Jesús Urueta, Balvino Dávalos, entre otros. Todos ellos ilustrados por el artista plástico Julio Ruelas" (15). A partir de esta semblanza es posible identificar el decadentismo como una modalidad en extremo del modernismo cuyos representantes fueron atenuando sus provocativos escándalos conforme dejaban la juventud o morían. No obstante, ante la mirada de varios colaboradores de Don Quijote, ese decadentismo no dejaba de ser parte de un modernismo extranjerizante y amoral.

${ }^{17}$ De acuerdo con la investigación realizada por Héctor Valdés, en esta revista se publicaba, en su mayoría, textos de franceses, que superaban en número los de escritores mexicanos.

${ }^{18}$ Para una historia más detallada sobre las implicaciones de este texto en torno a la autonomía regional y los parámetros filosóficos detrás de este, se sugiere revisar el artículo "Atenedoro Monroy: preceptiva y política en el fin del siglo XIX” de Leonardo Martínez Carrizales. 
asiduo traductor de Henry Wadsworth Longfellow, lo que revela cierta influencia romántica.

Otros ejemplos que nos permiten sostener el argumento de una animadversión por parte de Don Quijote hacia el modernismo de la Revista Moderna son algunas críticas aparecidas entre 1908 y 1909. En una de sus secciones, "Bibliografía", destinada a la crítica literaria, agregan un texto firmado con el pseudónimo Werther, en el que se analiza la colección de sonetos Troqueles antiguos, publicados por Ignacio Pérez Salazar y Osorio (Alidauro Zacintio), y se menciona lo siguiente:

Objeto de una vanagloria muy recomendable, y a causa de legítimo orgullo, era para los intelectuales de la ciudad de Puebla el no haber ido á dar de bruces en la ignominiosa red del modernismo, y á gala tenían el constituir un núcleo decididamente enemigo de todo escarceo y ambage de filiación "baudelairiana".

Asimismo, se menciona a Manuel Rivadeneyra, quien, según esta crítica:

tiene á menudo ciertos deslices del todo inconscientes, en los que se advierte una homeopática proporción de modernismo. Su predilección por el alejandrino francés le inclina todavía más del lado funesto, amén de cierto amaneramiento, cierta obscuridad y cierta difícil exposición que se notan en algunas obras de Manuel.

Sin embargo, el autor de la crítica afirma que ninguno ha pasado por el modernismo, incluso Alidauro, quien decide publicar su libro con versos alejandrinos:

Más, he aquí que dedica la séptima parte de su obra al "Arte moderno, al Arte nuevo, al modernismo"! . . . Si nuestro bueno y respetable amigo quiso torcer por la susodicha vereda del moderno decir, no lo ha conseguido, gracias á Dios. El escribir simplemente alejandrinos castellanos con sus catorce 
sílabas bien contadas 'al viejo modo,' con sus acentos oportunos, con el lenguaje de los que hablan castellano y las ideas sanas de las gentes cuerdas ... no es hacer "Arte Nuevo" ni modernismo, ni locura semejante. Y si no voy descarriado, adivino que "Alidauro" ha querido mofarse del modernismo en el último soneto de su libro, parodiando alguna composición "seria" del mismísimo Rubén Darío. (13-14)

Por otra parte, estadísticamente, por la predominante actualidad de los autores, la revista se asume contemporánea en el sentido en que, junto a los jóvenes vinculados al Colegio del Estado, también colaboran esporádicamente escritores provenientes del Seminario Palafoxiano (Federico Escobedo, Eduardo Gómez Haro, por ejemplo), así como personalidades extranjeras notorias en esos momentos, ya sea cultural o políticamente, aunque poco recordadas a la postre (Julio Florez, Manuel Ugarte, José Ingenieros, entre otros).

Estéticamente, se aprecia una adhesión a lo que se ha denominado "romanticismo tardío", una expresión vigente hacia la segunda mitad del siglo XIX en Europa e Hispanoamérica, en pos de la idealización de otras épocas; así como al realismo, con sus variantes como el costumbrismo. Además, a pesar de que en la nómina de Don Quijote aparecen personajes como Manuel Gutiérrez Nájera, Manuel José Othón o Manuel Santos Chocano, estos se retoman en su faceta de modernismo más atemperado y vigilante del rigor formal según la preceptiva clásica.

Para ilustrar lo anterior vale la pena resaltar una traducción publicada en el número 3 del tomo I, "Una página de Max Nordau". Se trata de un fragmento del ensayo escrito por Max Nordau, un crítico austrohúngaro, en 1893, Entartung, el cual sería traducido al español por Nicolás Salmerón y García en 1902 como Degeneración, y contaría con una amplia difusión y adhesión por sus ideas antidecadentistas en el arte. La traducción en la revista aparece firmada con el pseudónimo Gwymplaine ${ }^{19}$ y se trata de un fragmento del

${ }^{19} \mathrm{Al}$ cotejar esta traducción con la de Salmerón notamos pocas diferencias, 
capítulo "Diagnóstico". En estas páginas se presenta la idea de la producción artística individual y, además, se califica de degenerados y enfermos a quienes se establecen en grupos literarios, como los simbolistas:

El verdadero talento es siempre personal . . obedece á su impulso creador y no á una fórmula teórica predicada por el fundador de una nueva 'capilla' literaria o artística; desarrolla su obra en la forma que le es orgánicamente necesaria, y no en la que un jefe de secta le quiere imponer, por la única razón de que está en auge debido a un capricho de la moda. Basta que un escritor ó un artista se filie á un 'ismo' cualquiera, para que demuestre por este simple hecho su falta absoluta de personalidad, es decir de talento. (5)

Nordau, al criticar la nueva "capilla" y su "secta" como "capricho de la moda", tiene en la mira al simbolismo francés y su modalidad extrema decadente, que se extendió con rapidez hacia otros confines como Hispanoamérica. ${ }^{20}$ Este texto resalta no solo por las fuertes declaraciones que hace en torno al arte decadente de simbolistas y modernistas, ${ }^{21}$ sino también porque deviene de un idealismo, como se plantea en la introducción al número prospecto de Don Quijote, y de una fe en la propia creación. Refleja también la

lo cual nos podría llevar a especular que el uso del pseudónimo se debe a que no se trata de una traducción del original, sino de una versión modificada de la obra en español.

${ }^{20}$ De hecho, los posicionamientos de Nordau aparecen por primera vez en México en la primera época de la Revista Azul con la réplica inmediata de Rubén Darío.

${ }^{21}$ En una encuesta para la revista El nuevo Mercurio, editada por el guatemalteco radicado en París, Enrique Gómez Carrillo, Nordau tratará despectivamente a Darío, cuando imita a Verlaine, y al modernismo hispanoamericano: "El modernismo es la importación en España de modelos franceses, que ya no están de moda en Francia” (243). Será interesante anotar, para otro estudio, la relación que pudiera haber entre la publicación de Gómez Carrillo y Don Quijote, dada la nómina común de algunos colaboradores. 
animadversión que se consolida en distintas provincias hacia el decadentismo, la cual tiene que ver con la búsqueda de una literatura propia y fuera del centro.

Entre los artífices de Don Quijote existe el sentimiento del fin de siglo que demanda una obligada renovación de aquellos "hijos pálidos y tristes de este agitado fin de siglo" (Contreras 1). Por eso, en diversos textos se plantea el establecimiento de una literatura propia, tal como lo expresa Manuel Ugarte en uno de los textos publicados en la revista:

Lo que hemos hecho hasta ahora no ha sido en resumen mas que un arte colonial — colonial de Francia, colonial de España, colonial de Italia, - pero arte de reflejo, belleza que no tiene ninguna marca local, ni en los asuntos, ni en la inspiración, ni en la forma ... No hay razón para que la literatura siga siendo exótica, cuando tenemos territorios, costumbres y pensamientos que nos pertenecen . . . Nuestro pequeño caudal de aguas tiene que buscar lecho propio, en vez de sacrificarse y fundirse en el de los grandes ríos ... Porque entre nosotros todo es nuevo: la naturaleza, las pasiones, las costumbres, y pocas veces habrá recibido la literatura universal una contribución tan fecunda y tan vasta. (4-5)

La idea anterior parece relucir en todos los números de Don Quijote, pues se reitera la necesidad de una literatura de México, y este texto del argentino Ugarte sirve para ejemplificar uno de los objetivos de la revista.

Estas ideas coincidían en mucho con los cambios que la misma institución de educación superior en Puebla había tenido a partir de la primera década. De acuerdo con Herrera Feria:

En el ámbito de la educación superior, los últimos años del siglo XIX y la primera década del siglo XX significaron un período de fructífera renovación de los estudios y cátedras y de aliento a las observaciones científicas, pero también de adaptación a los requerimientos de las nuevas actividades produc- 
tivas: las enseñanzas prácticas que capacitaban para el trabajo en el corto plazo ganaron terreno, los cursos de telegrafía práctica y los que preparaban para las actividades comerciales ganaron el favor del público y tuvieron mayor demanda, mientras las largas carreras profesionales, como la medicina y la abogacía, vieron mermar su matrícula. (202)

Esto significó un aumento considerable en la matrícula de estudiantes, además de la inscripción formal, por primera vez, de mujeres entre 1901 y 1902 (Huerta Jaramillo 6). Dichos factores determinaron que la población estudiantil tuviera un impacto directo en la ciudad y sociedad de la época. Así es como Don Quijote no representaba solamente las funciones de una revista de arte estudiantil, sino, como lo ha dicho Esparza, de un "atalaya de luz" que influyó decididamente en la época. Por ello la manifiesta preocupación de los jóvenes humanistas ante el pragmatismo que se impuso durante la década, al observar cómo la preferencia de formación profesional se decantaba hacia las actividades comerciales en lugar del conocimiento profundo. Detrás de dicha angustia se percibe una profunda posición ideológica que empata con la situación política y cultural en los últimos años de la primera década del siglo XX.

Márquez Carrillo señala que, a partir de la reforma del Colegio de Estado mandatada en 1893 por el gobernador de Puebla, Mucio P. Martínez, se consolidó, desde 1895, un grupo político e intelectual que reguló no solamente la orientación educativa de dicha institución sino también de otros centros educativos como la Escuela Normal para maestros, desde donde se estableció una nueva política educativa y cultural. Entre los miembros destacados se encontraban Miguel Serrano, Rafael Serrano y José Rafael Isunza, director del Colegio del Estado desde 1893 hasta 1910. Resalta además que:

Su filiación era el krauso-positivismo, una corriente de pensamiento que, oponiéndose al positivismo porque no proporcionaba a los estudiantes valores trascendentes, se propuso conciliar la fe cristiana con el uso del método científico, el 
desarrollo de la ciencia, el dominio de la técnica y el pensamiento político moderno. (Márquez Carrillo 96)

Frente al predominio del positivismo en México se encontraba el liberalismo añejo que había inspirado la Constitución de 1857, el cual, para mantener la vigencia de sus ideales cada vez más lejanos, apeló, según describe Sánchez Cuervo, al krausismo: "Podemos decir que el krausismo de Tiberghien fue empuñado contra el positivismo oficial por un liberalismo disidente y desplazado" (23-24). Específicamente, en Puebla este campo de luchas derivó en una suerte de alianzas interesantes:

Hacia finales de la primera década del siglo XX, dado el desarrollo de las sociedades metodistas, la presencia del krausismo y el surgimiento de la doctrina social de la iglesia católica, podemos ver en la ciudad de Puebla toda suerte de alianzas contra el positivismo oficial en los principales centros educativos: el Instituto Normal del Estado, la Universidad Católica Angelopolitana, el Colegio del Estado y los institutos de la Iglesia Metodista. (Márquez 91)

Por lo tanto, en la lectura de los diversos números de Don Quijote resulta interesante descubrir las colaboraciones de José Rafael Isunza, Rafael Serrano, Federico Escobedo, Manuel Rivadeneyra y Palacio como la base de un grupo político e intelectual diverso (krausistas-positivistas y católicos sociales) que fue preparando a la juventud para lo que acontecería de manera más clara a partir de 1910. En ese sentido, la revista puede comprenderse como el espacio desde el cual se acumula cierto capital cultural cuyos réditos se cobrarán en el campo político a partir de las elecciones presidenciales de 1910, el fraude electoral y la llegada a la presidencia de Francisco I. Madero.

Aunque esta fuerza relacional con lo político no es del todo evidente en los textos publicados en Don Quijote, sí resulta visible en las relaciones de colaboración entre escritores autoproclamados revolucionarios, serdanistas y maderistas que escribían en sus páginas. Siguiendo a Márquez, debemos considerar que los principales artí- 
fices de la revista en realidad formaban parte de un grupo de estudiantes favorecidos por el grupo con el poder educativo y cultural:

Tradicionalmente uno de los espacios de los que se aprovechaban los universitarios para introducirse en las lides políticas era la Junta Directiva de los Estudiantes del Colegio del Estado. Esta organización funcionaba como un trampolín para escalar posiciones de poder. Entre 1905 y 1910 estuvieron en la dirección de la misma Alfonso G. Alarcón (1884-1953), Luis Gonzaga Quintana (1888- ), Gil Jiménez Aguilar (1883-) y Luis Sánchez Pontón (1889-1969), todos ellos pertenecientes o vinculados con importantes grupos de la sociedad y la cultura de Puebla. (99)

En la lista se distingue a dos fundadores de Don Quijote: Alarcón y Jiménez. En un principio, el campo de acción de la revista era lo cultural a través de la difusión de una literatura que apelaba a una amplia tradición clásica en Puebla durante el siglo XIX, ${ }^{22}$ la cual debía renovarse en lo espiritual como principio para una formación moralmente íntegra. Lo anterior explica el corte "tardo romántico" o mesuradamente modernista, así como el privilegio de una lírica intimista o del cuento realista en muchas colaboraciones durante la vida de la publicación.

En 1910, ante los comicios para presidente, la participación estudiantil se vuelve más activa políticamente. Los redactores de Don Quijote, al lado de otros grupos estudiantiles, se sumaron al club antirreeleccionista de Aquiles Serdán. El maderismo cobró mayor auge en el Colegio del Estado, de tal manera que "La Junta Directiva de los Estudiantes del Colegio del Estado se pronunció por participar en el recibimiento a Madero como candidato a la Presidencia de la República que tendría lugar el 15 de Mayo, llevando la bandera del Colegio, pese a la oposición de los estudiantes pensionados y

${ }^{22}$ Los antecedentes más claros son Manuel Carpio, Alejandro Arango y Escandón, Manuel Pérez Salazar y Vengas, y Tirso Rafael Córdoba. 
los hijos de funcionarios públicos" (Márquez 102). Además, en las siguientes reuniones se comisionó a Alarcón como el orador oficial del evento. Tras el fraude electoral del 7 de julio se organizó una manifestación popular que, entre otros acontecimientos, concluyó en una fuerte represión estudiantil y el encarcelamiento de Alarcón y Jiménez. Tal inestabilidad social provocó la destitución del gobernador de Puebla y el nombramiento de Isunza, quien ya había renunciado a la dirección del Colegio del Estado como protesta por la represión contra estudiantes.

Alarcón, tras ser liberado de los cargos que tenía en contra, participó en el Primer Congreso Nacional de Estudiantes, celebrado en septiembre de 1910, junto con Luis Quintana, ${ }^{23}$ derivado de lo cual no solamente expresaron la necesidad de una reforma educativa autónoma y menos centralizada, sino que establecieron vínculos con otros estudiantes de la República opositores al régimen porfirista y se estrechó la relación con Alfonso Reyes ${ }^{24}$ cofundador del Ateneo de la Juventud. Estos acontecimientos permiten avizorar el rumbo que tomaba la revista hacia la mitad de su vida y también vincular este proyecto editorial con los propósitos de la juventud intelectual mexicana. Como menciona Álvaro Matute en El Ateneo de México:

Fueron gente que en sus años estudiantiles dejó el destrampe juvenil por la lectura. Todo lo que hicieron de mayor significación tiene en el libro el eje de sus preocupaciones, si se exceptúa a los cuatro pintores y a los tres concertistas, quienes empero, no fueron ajenos a la palabra impresa. La lectura llevó a los del Ateneo a la creación y a la política. No sólo enseñaron y divulgaron, sino que también se expresaron en obra escrita y en el ágora revolucionaria.

${ }^{23}$ Presidente de la Junta Directiva de la Escuela Preparatoriana del Colegio del Estado.

${ }^{24}$ Evidencia de ello queda, por ejemplo, en la recopilación de correspondencia entre Reyes y Rafael Cabrera, que Serge I. Zaïtzeff realizó bajo el título Alfonsadas: correspondencia entre Alfonso Reyes y Rafael Cabrera, 1911-1938, publicado por el Colegio Nacional en 1994. 
El papel político-cultural de los ateneístas fue fundamental, pues encabezaron el programa educativo y cultural para la segunda década del siglo XX. Como dice Enrique Krauze en Caudillos culturales en la Revolución mexicana (1990):

La renovación por la que propugnaban los ateneístas, en crítica abierta del positivismo oficial, tenía un carácter similar al de la apertura maderista. Una nueva generación intelectual también quería desplazar a la gerontocracia cultural gobernante, desplazarla de sus puestos y de su ideología, y modernizarse. (51)

Esta inquietud se expresó pronto en la efímera revista Savia Moderna (1906), la cual, no nos queda duda, habrá servido como modelo para Don Quijote pues, a pesar de estar posicionada como uno de los órganos difusores del modernismo y del futuro ateneísmo, en ella se retomaba el Siglo de Oro y se proponía la búsqueda de nuevas formas poéticas, teniendo como epítomes a Manuel Gutiérrez Nájera y Manuel José Othón. Esta relación, con sus altibajos en el campo literario, significó una plataforma de impulso fundamental tanto para Alarcón como para Cabrera, quienes, al abandonar el proyecto editorial y concluir sus estudios de licenciatura, fueron incorporándose al Ateneo de la Juventud. ${ }^{25}$

Por ejemplo, para Cabrera, la adhesión al Ateneo implicó el despunte en su actividad profesional, pues como ha reseñado Blázquez:

[En 1916] Julio Torri recibiría a Rafael Cabrera en el Departamento de Conferencias y Propaganda en la Dirección Ge-

${ }^{25}$ Este dato lo ha retomado Blázquez a partir de la revisión de Correspondencia I, 1907-1914, de Alfonso Reyes y Pedro Henríquez Ureña, donde se menciona el "descubrimiento" de Rafael Cabrera y Alfonso G. Alarcón y su incorporación al Ateneo de la Juventud. No obstante, como hemos de referir con mayores datos más adelante, el contacto inicial entre ellos se debió a la polémica causada en 1907 por la segunda época de la Revista Azul a cargo de Manuel Caballero donde la "Juventud literaria de Puebla" hizo contrapeso a la protesta ateneísta. Queda en la incertidumbre si el olvido de Reyes es premeditado. 
neral de Bellas Artes. Allí frecuentaría a Mariano Silva y Aceves, Carlos Días Dufoo Jr., Jorge Enciso, Saturnino Herrán, Ramón López Velarde, Genaro Estrada, Manuel Toussaint, Efrén Rebolledo y, eventualmente, a Enrique González Martínez, el poeta que había torcido el cuello al cisne rubendariano convocando al búho de Minerva. En 1918 ingresaría a la Secretaría de Relaciones Exteriores para, al año siguiente, ser nombrado Segundo Secretario en Roma.

La trayectoria de Alarcón y Cabrera para vincularse con los ateneístas se encuentra dibujada por una serie de altibajos en el campo literario donde, en algunas ocasiones, se ubican como fuerzas contrarias. Quizá esto explique la limitada colaboración de ateneístas en Don Quijote. Pedro Henríquez Ureña se publica en dos ocasiones: primero, en septiembre de 1910, con un texto donde aborda el libro Profesores de idealismo de Francisco García Calderón, leído en sesión del Ateneo de la Juventud de México; y después, en 1911, con un ensayo sobre la vida de Oscar Wilde. En el mismo número de septiembre de 1910, aparece un texto de Julio Torri, también leído en la sesión del Ateneo, titulado "Diálogo de cinco murmuradores", dedicado a Antonio Caso, el cual también se publicará posteriormente, en 1911, en Revista de Revistas.

Don Quijote conformó, como hemos dicho, una red de revistas de provincia. La primera revista con la que entabla diálogo es Fausto, de Cuautla, Morelos. También se relaciona con Kalendas, de José Becerra y Mariano Azuela, publicada en Lagos de Moreno, Jalisco; Alborada, que, aunque se menciona que es de Saltillo, Coahuila, se ha hallado que fue publicada también en Lagos de Moreno ${ }^{26}$; Capullos, de San Luis Potosí, de Agustín Vera y Jorge Adalberto Vázquez; El entreacto, que incluye en sus páginas un artículo dedicado a Don Quijote, que se reproduce en el número 8, tomo I de la revista poblana, en donde se menciona que "subscripciones como ésta, para

${ }^{26}$ Esto se ha determinado al consultar la tesis "Escritores de una ciudad encantada. El grupo literario laguense de 1903” de Irma Estela Guerra Márquez. 
sostener con ella la vida y el lustre de un paladín del ideal, de un órgano de civilización y de refinamiento intelectuales, eso no se ve entre nosotros, sino una vez cada siglo" (15).

Esta red entre revistas culturales de provincia permite reconocer la constante comunicación y conocimiento de un movimiento literario poco estudiado hasta ahora, que se gestaba paralelamente a lo que acontecía en la Ciudad de México. Esto provocó una lucha de fuerzas y una dinámica capital-provincia que, en materia literaria, aparece profundamente reflejada, por ejemplo, en la obra poética de Ramón López Velarde. Es interesante establecer como denominador común entre el poeta jerezano y Don Quijote a Eduardo Correa, director de la revista La provincia y el periódico El observador, de Aguascalientes. Correa aparece en el índice de la revista en cinco ocasiones, con poesía propia, pero además fue un personaje decisivo para la trayectoria literaria de López Velarde en sus años de estudiante en el Instituto de Ciencias de Aguascalientes ${ }^{27}$ (1906-1907).

Tanto Becerra, artífice de Kalendas, como Correa se posicionaron en torno a la conformación de una literatura fuera del centro. El primero, en un discurso que pronuncia en su ciudad, recalca que la producción de periódicos literarios en las provincias, durante los primeros años del siglo XX, fue más prolífica que en la metrópoli (en Guerra Márquez 93). El segundo, Correa, poeta modernista que mantuvo una relación estrecha con Manuel Gutiérrez Nájera, colaboró en varios números después de haber enviado su libro Oropeles a la redacción de Don Quijote, obra de la cual se escribió lo siguiente:

"Oropeles" nos visita precedido de muy envidiable fama, pues le hemos visto ensalsado sin medida por la prensa del

${ }^{27}$ En el estudio introductorio que Alfonso García Morales dedica a la Obra poética de López Velarde, escribe el siguiente dato de la atención que Correa prodigó a Bohemio, revista del poeta y sus compañeros de instituto: "Sin duda estaba decidido a ganárselos para la causa, especialmente a López Velarde, el más predispuesto y prometedor, a quien enseguida abrió las puertas de sus publicaciones y al que, durante los años siguientes, arrastró a aventuras literarias y políticas poco afortunadas" (14). 
país. La provinciana, se entiende, que bien se conocen los puntos alcanzados por el donoso desprecio con que se ven por la metrópoli nuestros sanos esfuerzos en cualquier sentido. (16)

De estas declaraciones se trasluce un posicionamiento en defensa de la producción literaria fuera de la capital mexicana, a través de una red de revistas publicadas en distintas ciudades de provincia. ${ }^{28}$ Esta posición tiene un claro antecedente en la polémica que se desató cuando Manuel Caballero inició la segunda época de la Revista Azul de Gutiérrez Nájera, en 1907, declarando la guerra abierta contra el decadentismo.

En contra de Caballero y su programa se alzaron los jóvenes ateneístas y colaboradores de Revista Moderna y Savia Moderna; a favor se redactaron pronunciamientos de Bohemio, la revista de López Velarde; El Observador, de Correa; y la "Juventud literaria de Puebla", integrada por Atenedoro Monroy, Rafael Cabrera y Alfonso G. Alarcón. ${ }^{29}$ Se trata de un capítulo en la literatura mexicana que, pese a contar con las fuentes directas para su estudio, los textos, ha sido poco puesto en su justa dimensión. ${ }^{30}$ Para la historiografía

${ }^{28}$ Dentro de este campo de fuerzas resulta relevante considerar que el término peyorativo "literatura provinciana" surge como resultado de esta relación de fuerzas donde el capital simbólico que acumula la producción generada desde la capital del país, permeada por un centralismo político y económico predominante, permite desdeñar todas aquellas manifestaciones literarias ajenas o distintas. Este fenómeno, que no es exclusivo de México, tiene su origen en la avanzada capitalista del siglo XIX, que utilizó a las grandes ciudades modernas como el centro económico y político de naciones y del mundo, de donde además partía un programa ideológico a modo (revisar, por ejemplo, París, capital del s. XIX de Walter Benjamin o La ciudad letrada de Ángel Rama).

${ }^{29}$ Previamente en El Entreacto, un bisemanario de espectáculos, literatura y arte, Eduardo Gómez Haro, Guillermo Fernández de Lara y Federico Escobedo habían publicado unas "Contraprotestas a favor de la Revista AzuP".

${ }^{30} \mathrm{Al}$ respecto debemos reconocer los siguientes estudios con mayor amplitud de perspectiva: la nota de Leonardo Martínez Carrizales, "Una interpretación literaria de la Revista Aそul, segunda época", a propósito de la edición facsimilar que preparó Fernando Curiel sobre la publicación; "Últimas batallas sobre el modernismo: la segunda Revista Azul de México" de Alfonso García Morales, en Biblio- 
literaria mexicana, reconstruida por algunos de los participantes en la polémica, como Alfonso Reyes, se trató de un combate entre el provincialismo añejo y neoclásico de provincia contra el pujante cambio progresista de la capital, entre preceptistas conservadores de formas literarias contra portavoces del arte libre, finalmente entre católicos y liberales. Aunque esta polarización iría atenuándose de manera paulatina conforme entraba el conflicto armado de la Revolución mexicana, dejó un resabio que ha costado caro a la historia y crítica de la literatura mexicana de la primera década del siglo XX.

Lo que era una posición política — recordemos, por ejemplo, la intervención de Luis Quintana en el Primer Congreso Nacional de Estudiantes (1910), quien rechaza las intenciones gubernamentales de centralizar la administración de la enseñanza - se trasminaba también al campo literario. El modernismo, en la modalidad extrema del decadentismo, era incómodo para las ideas literarias en diversos puntos de la República, pero estos lo fueron asimilando gradualmente con el paso de la primera década, en parte por la madurez y control que el Ateneo tomó como grupo cultural y político dominante. Como resabio de esta lucha de fuerzas quedó un centralismo de la Ciudad de México que opacó al resto de las opciones literarias del momento. Por ello, en este estudio insistimos en una lectura directa de las fuentes, para lo cual los índices y ediciones facsimilares de revistas literarias de aquella época resultan fundamentales. En el caso particular de Don Quijote, podemos concluir, tras señalar el contexto general y trascendencia en su momento, que no solamente sirvió como "atalaya de luz" para la cultura en Puebla en los albores del siglo XX, sino que resumió en su programa de publicaciones una posición intelectual derivada del krauso-positivismo, que buscaba renovar tanto el fracaso de los ideales liberales plasmados en la Constitución de 1857 como el decaimiento de la dictadura porfirista.

teca Virtual Miguel de Cervantes; y La construcción del modernismo, antología de textos preparados y estudiados por Belem Clark de Lara y Ana Laura Zavala Díaz. 
Paralelamente a la juventud del Ateneo en la Ciudad de México, en Don Quijote confluyó una nueva generación en busca de formas expresivas particulares. Si bien las diferencias parecieron polarizarse en un primer momento debido a la polémica causada con la aparición de una segunda época de la Revista Azul, en ambas maneras aparecía un afán renovador de las letras. En este sentido, el modernismo no era el mismo en la ciudad de Puebla que en otras regiones, aunque sí existía un interés por publicar a escritores jóvenes, a una nueva generación trastocada por los cambios paradigmáticos, no solo tecnológicos, del "progreso", como se diría en esos momentos, sino también literarios e incluso de educación.

Lamentablemente, el centralismo cultural acabó por opacar y denostar a la mayoría de los proyectos culturales gestados desde provincia. Pero ahora que, con la suficiente distancia temporal, puede leerse de manera más crítica el modernismo literario, es posible retomar ciertos matices plenamente justificados por las circunstancias históricas y políticas del momento para enriquecer el amplio y diverso panorama de la literatura mexicana. Sirva este caso de estudio de la revista Don Quijote para abrir nuevos estudios y conocimientos.

\section{Bibliografía}

Blázquez Espinosa, José Carlos. "Un ateneísta olvidado”. Proceedings of the 38th Instituto Internacional de Literatura Iberoamericana Internacional Congress: Independencias: Memoria y Futuro, editado por Gwen Kirkpatrick y Enrique Cortez, Georgetown University, 2010.

Bourdieu, Pierre. Las reglas del arte. Génesis y estructura del campo artístico. Anagrama, 1995.

"El campo literario. Prerrequisitos críticos y principios de método". Criterios, no. 25-28, (enero 1989-diciembre 1990), pp. $20-42$.

Contreras, Felipe T. "Inocencio". Don Quijote, tomo I, no. 1, 1908, pp. 1-3.

Cordero y Torres, Enrique. Historia del periodismo en Puebla (18201946). Ediciones de Bohemia Poblana, 1947. 
Don Quijote. Revista Mensual de Arte. Dirigida por Rafael Cabrera y Alfonso G. Alarcón, Puebla, 1908-1911.

Englekirk, John E. "La literatura y la revista literaria en Hispanoamérica". Revista Iberoamericana, vol. XXVI, no. 51, enero-junio 1961, pp. 9-80.

Herrera Feria, María de Lourdes. "El Colegio del Estado de Puebla y el Primer Congreso Nacional de Estudiantes en 1910". Revista Mexicana de Historia de la Educación, vol. V, no. 10, 2017, pp. 199221, doi: https://doi.org/10.29351/rmhe.v5i10.114

Huerta Jaramillo, Ana María Dolores. "Los estudiantes poblanos en 1910". Lecturas Históricas de Puebla 100, Secretaría de Cultura, 1992.

López Velarde, Ramón. Obra poética (verso y prosa). Edición, estudio introductorio y notas por Alfonso García Morales, Universidad Nacional Autónoma de México, 2016.

Matute, Álvaro. El Ateneo de México. Fondo de Cultura Económica, 1999, colección Fondo 2000, bibliotecadigital.ilce.edu.mx/ sites/fondo2000/vol2/25/htm/portada.htm

Márquez Carrillo, Jesús. Educación, historia y sociedad en Puebla. Raices, tiempos, huellas. BUAP, 1999, Cuadernos del Archivo Histórico Universitario, Benemérita Universidad Autónoma de Puebla.

Nordau, Max. Degeneración. Tomo Primero, traducido por Nicolás Salmerón y García, Librería de Fernado Fé Saez de Jubera, Hermanos, 1902.

. "El modernismo en España y América". El nuevo Mercurio, no. 3, marzo 1907, pp. 243-244.

“Oropeles". Don Quijote, tomo I, no. 2, 01 marzo 1908, p. 16.

Leyva, José Mariano. Perversos y pesimistas: los escritores decadentes mexicanos en el nacimiento de la modernidad. Tusquets editores, 2013.

Reyes, Alfonso y Pedro Henríquez Ureña. Correspondencia I, 19071914. Edición, introducción y notas por José Luis Martínez, Fondo de Cultura Económica, 1986.

Sotelo Mendoza, Humberto. "La revolución maderista en Puebla y los estudiantes del Colegio del Estado". Tiempo Universitario, año 2, no. 11, 10 junio 1999, 148.228.11.41/archivo-2019/sites/default/files/Tiempo\%20Universitario/1999/num11/index.html 
Ugarte, Manuel. "La orientación actual”. Don Quijote, tomo III, no. 6, marzo 1910, pp. 1-5.

Werther [seudónimo]. "Bibliografía”. Don Quijote, tomo 2, no. 1, 1 diciembre 1908, pp. 13-14. 